\title{
El primer año de la Corporación RTVE: cambios en la programación de La 2
}

\author{
Erika FERNÁNDEZ GóMEZ \\ Universidad Internacional de La Rioja (UNIR) \\ erika.fernandez@unir.net
}

\begin{abstract}
Resumen
Este artículo presenta la programación del segundo canal de la televisión pública española durante el primer año de la Corporación RTVE (2007) y el último del Ente Público RTVE (2006). El cambio de organismo supuso el intento de que La 2 fuera más competitiva ya que, hasta el momento se había posicionado como una televisión para minorías. Se ha llevado a cabo un análisis cualitativo de la parrilla y un estudio cuantitativo de la programación con la finalidad de determinar la naturaleza de los contenidos. El objetivo ha sido comprobar las modificaciones llevadas a cabo, dejando constancia de las diferencias programáticas y la evolución entre el Ente Público y la Corporación.
\end{abstract}

Palabras clave: Televisión pública; La 2; Corporación de Radio y Televisión Española; Ente Público RTVE; programación y reestructuración.

The first year of the Spanish Radio and Television Corporation: changes in the schedule of the second channel La 2

\begin{abstract}
This paper shows the programming of the second channel during the first year of Spanish Radio and Television Corporation (2007). It also presents the contents of the last year of RTVE Public Body (2006). The change of the organization turned on the attempt of get La 2 to be more competitive. Until the moment, the second channel was characterized by being the television of minorities. It was carried out a qualitative analysis of the schedule and a quantitative study of the programming in order to find the nature of the programmes. The main goal is to check the changes between the Public Body and the Corporation.
\end{abstract}

Key Words: Public television; La 2; Spanish Radio and Television Corporation; RTVE Public Body; schedule and restructuring.

\section{Referencia normalizada:}

Fernández Gómez, E. (2013) El primer año de la corporación RTVE: cambios en la programación de La 2. Historia y Comunicación Social. Vol. 18 Nº Especial Octubre. Págs. 723-736.

Sumario 1. Introducción 2. Metodología 3. La $2^{\mathrm{a}}$ Cadena de TVE: de una minoría a una inmensa mayoría 4. Del Ente a la Corporación 4.1. Análisis de la parrilla de programación 4.2 Naturaleza de los contenidos 4.2.1 Tipos de programas de lunes a viernes 4.2.2 Tipos de programas de entretenimiento 4.2.3 Tipos de programas en el fin de semana 4.3 Tipo de producción 4.4 Audiencia 5. Conclusiones 6. Referencias bibliográficas 7. Notas 


\section{Introducción}

Desde sus inicios a mediados de los años 60, el segundo canal de la televisión pública española se consideró una televisión para minorías, aburrida, y expresamente cultural que no figuraba entre las opciones de la audiencia. A la ruptura del monopolio de la televisión pública en los 90, con la implantación de los tres primeros canales de televisión privada (López-Vidales, Azurmendi-Adarraga y Ortiz-Sobrino, 2012: 57) se sumó en el inicio siglo XXI la oferta de televisión por cable, por ADSL y la Televisión Digital Terrestre. Este incremento de la oferta amplió la competencia y las distintas posibilidades de ver la televisión. Como consecuencia, desde el año $2000 \mathrm{La}$ 2 perdió audiencia progresivamente (Sofres, 2008).

En este contexto de cambios en el panorama audiovisual, se puso en marcha la Corporación RTVE (2007). A través de la Ley 17/2006, de la Radio y la Televisión de Titularidad Estatal se intenta acabar con la dependencia de la televisión pública mediante órganos de gestión menos dependientes de los gobiernos y partidos políticos y con un mayor control de la gestión y objetivos de servicio público (López Cepeda, 2012: 20). La nueva normativa derogaba el anterior Ente Público, regulado mediante la Ley 4/1980, de 10 de enero, del Estatuto de la Radio y la Televisión.

La transformación del ente público en este organismo vino acompañado de diferentes novedades y entre ellas la que concierne a este estudio: La 2 se renueva. El 16 de abril de 2007, el segundo canal de TVE puso en marcha su nueva parrilla de programación primaveral con la que se pretendía dotar a la cadena de una identidad nueva. Es decir, manteniendo lo que la caracteriza como son los espacios de servicio público y entre ellos, los documentales y programas de cultura, pero al mismo tiempo, buscando una renovación de cara a ser más competitivos y ganar audiencia.

De manera que, se ha considerado importante hacer una aproximación a los contenidos de la $2^{\mathrm{a}}$ Cadena de Televisión Española en este período de modificaciones. Para ello se ha realizado un análisis de la programación de La 2 a lo largo de dos temporadas, tanto antes como después del anuncio de la reestructuración, con la finalidad de detectar los cambios que se hayan producido.

Por último, tras 54 años de vida, en 2010 la televisión pública estrenó un nuevo sistema de financiación ${ }^{1} \mathrm{y}$, por primera vez desde su nacimiento, dejó de emitir publicidad. De manera que esta investigación, además de recoger las diferencias programáticas experimentadas por la cadena con el cambio de normativa, deja constancia de un estudio de la programación de La 2 en la que se incluyen espacios comerciales.

\section{Metodología}

El estudio se fundamenta en la recogida de información documental sobre los contenidos programados por La 2 y que corresponden a dos temporadas objeto de estudio $^{2}$. El segundo trimestre de 2006 permite conocer las características de progra- 
mación de la cadena un año antes del anuncio del cambio y el segundo trimestre de 2007, corresponde a la temporada en la que se anuncia la transformación de la cadena. Se ha tomado como referencia una semana de programación teniendo en cuenta que las parrillas se construyen de lunes a domingo ${ }^{3}$.

El hecho de que la $2^{\text {a }}$ Cadena de TVE sea una cadena pública condiciona el marco teórico de la investigación. A la hora de plantear el estudio de los contenidos de la cadena se tomaron como referencia los principios que rigen la programación de la televisión pública en España y que están recogidos en la Ley 17/2006 y publicados por el Instituto Oficial de Radio Televisión Española de forma más amplia y detallada (IORTV, 2007: 13-21). Así, se establece que la producción propia debe tener un peso sustancial en el conjunto de la programación de RTVE y el deber de apoyar de manera activa a la industria audiovisual nacional. Se recoge la prioridad absoluta que debe dársele a la información como elemento distintivo de la Corporación y que tendrá un papel activo en la divulgación del conocimiento. Además, se velará por la difusión y protección de sectores específicos de la cultura española como la música. También se incluye el dar cobertura a los acontecimientos deportivos de mayor trascendencia, sin olvidar el seguimiento de los deportes minoritarios.

Así, por un lado se ha abordado la parrilla desde una perspectiva cualitativa desde la que se pretende observar, describir y explicar la programación de La 2 a través de un elemento clave: la construcción de la rejilla. Se ha procedido a comparar la programación de la cadena durante la etapa del Ente Público y bajo el mandato de la Corporación. Por otro lado, se ha empleado la metodología cuantitativa con la finalidad de determinar la naturaleza de los contenidos (informativos, formativos o de entretenimiento) y el origen de la producción (propia, ajena, derechos de emisión, externa y coproducción). Se ha tenido en cuenta la clasificación de tipos de programas realizada por Carles Marín (2006:77-100). Así como las clases de producción establecidas por Cortés (2001: 108-109).

\section{La $2^{\mathrm{a}}$ cadena de TVE: de una minoría a una inmensa mayoría}

TVE-2, conocida por su nombre publicitario La 2 e históricamente por el $\mathrm{UHF}^{4}$, es la segunda cadena de servicio público de titularidad estatal en España. Se inauguró el 1 de enero de 1965 y la programación regular comenzó el 15 de noviembre de 1966. Pero no fue hasta 1982, con la llegada del mundial de fútbol, cuando tuvo una cobertura de dimensión nacional. El Ministro de Información y Turismo, Manuel Fraga, decidió que Televisión Española pusiera en marcha, al igual que hacían otras cadenas europeas, un segundo canal de televisión que emitiese bajo la tecnología del UHF. A partir de los primeros años sesenta, los organismos públicos contaron con las segundas cadenas y establecieron una complementariedad coordinada y armónica entre las dos programaciones. De modo que hicieron una oferta con un fuerte sesgo cultural 
que iba dirigida a las minorías urbanas. Pues era absurda una hipotética competencia entre los dos canales de servicio público (Contreras y Palacio, 2003: 57).

Con la llegada de las televisiones privadas se produjo un proceso de renovación en TVE-2. Desde 1990 pasó a llamarse La 2 y el proceso concluyó a mediados de 1991, cuando cambió por completo su identidad corporativa. Bajo la nueva denominación se procuró enfatizar los contenidos culturales del canal, acercar las franjas de la cadena a públicos minoritarios y apostar por espacios más arriesgados mientras que, La Primera, asumiría un enfoque generalista. Tendría como lema: "La 2, para una inmensa minoría". Si bien, Gómez-Escalonilla (1998: 234-273), señalaba en su tesis doctoral del año 1998 que, en el período de tiempo que la segunda cadena llevaba emitiendo siempre ha tenido la finalidad de entretener al público. La segunda funcionalidad del canal ha sido formar, y la tercera, informar.

Durante la última etapa del Ente Público RTVE, La 2 se caracterizó por ser la quinta preferencia de la audiencia, solo por delante del incipiente canal La Sexta. Como se puede ver en el Gráfico 1, en seis años perdió un 3,1\% de cuota de mercado al pasar del 7,9\% al 4,8\%.

Ante esta situación, uno de los objetivos para el nuevo presidente de la Corporación $^{5}$, Luis Fernández, fue la recuperación del segundo canal ${ }^{6}$. Así, bajo el eslogan $\mathrm{La} 2$ Se Mueve se ponía en marcha el proceso de reestructuración de la cadena. El director de TVE, Javier Pons, afirmaba: "queremos que La 2 sea diferente, pero potente. Ser los mismos pero llegar a más público, dotar a la cadena de una estrategia propia. Una televisión para todos, pero con compromiso, donde, además de ganar espectadores, estén representadas todas las sensibilidades que no se ven reflejadas en la Televisión en estos días" (Gabinete de Prensa de RTVE, 2007).

Gráfico 1. Evolución de la audiencia de la televisión generalista en España (20002006)

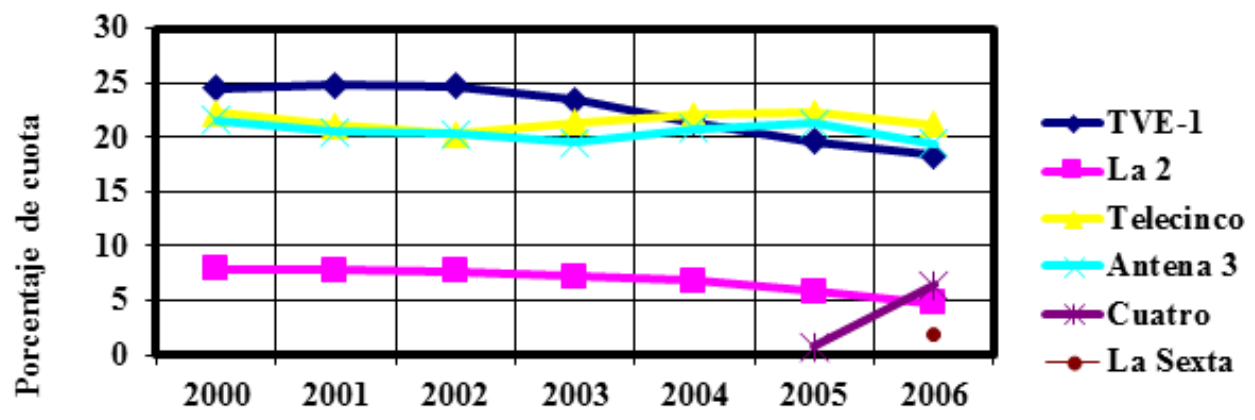

Años

Fuente: Taylor Nelson Sofres Gráfico: Elaboración propia 


\section{Del ente a la corporación}

En este apartado se presentan las modificaciones llevadas a cabo en la programación de La 2, dejando constancia de las diferencias programáticas y la evolución entre el Ente Público de Carmen Caffarel (directora general) y la Corporación.

\subsection{Análisis de la parrilla de programación}

Comparando la rejilla que la cadena presentaba en junio de 2006 y de 2007, se aprecia como la etapa del cambio se caracteriza por una parrilla de programación en la que se ha procedido a reestructurar los contenidos ya existentes. Pues se han desplazado de franja horaria o se han ampliado o reducido su emisión.

Por ejemplo, el programa que ofrece conciertos en directo de artistas del panorama musical actual emitidos en Radio Nacional (Los Conciertos de Radio-3), se emitió un año antes de los cambios de lunes a jueves en las bandas horarias de madrugada y segunda madrugada. En la parrilla correspondiente a la renovación de la cadena el espacio quedó reducido a una única emisión semanal (la segunda madrugada de los jueves).

Otro de los espacios que de un año a otro cambió no sólo la periodicidad sino también el formato ha sido Miradas 2. Una de las estrategias planteadas para la nueva programación de la cadena fue la absorción de este informativo cultural por el noticiario de carácter generalista La 2 Noticias. De manera que este programa continuó emitiéndose diariamente pero su duración estuvo condicionada por las noticias del día. Por otro lado, esto se compensó con su programación como espacio autónomo en la madrugada del viernes. Sin bien, en 2006 la actualidad cultural se ofrecía diariamente y en la franja de máxima audiencia pues se emitía de lunes a viernes antes del Sorteo de la Bonoloto y el informativo de la noche. De modo que gozaba de una mejor ubicación en la parrilla al constituir un programa de acceso a los espacios fuertes de la noche.

Las principales permutas para hacer más competitiva a la cadena se aprecian en la franja de máxima audiencia. En 2006, La 2 presentaba una oferta menos competitiva con la emisión de espacios de servicio público que siempre han caracterizado a La 2 como demasiado cultural y, por lo tanto, para minorías. Este es el caso de Off Cinema (cine independiente) o Carta Blanca (programas culturales). En 2007, aunque se mantuvieron espacios insignia como Documentos TV o Versión Española (cine nacional), se programaron series de reconocimiento internacional como Mujeres Desperadas o Caso Abierto con el objetivo de ganar espectadores. Asimismo, en la etapa del cambio el prime time comenzó una hora antes con la finalidad de adelantarse a la competencia, Así, si con el Ente el segundo canal programaba sus platos fuertes de la noche a las 22.30 horas (media hora después que la competencia), con la Corporación lo hizo a las 21.30 horas.

Por otro lado, el paso del Ente a la Corporación supuso la eliminación de cuatro espacios vinculados al servicio público de la cadena: dos franjas diarias de progra- 
mación territorial (mediodía y acceso a prime time); la emisión diaria de deportes tras el informativo territorial vespertino y el magazín $i P O P$ sobre actualidad musical, videoarte o juegos. En el lugar de estos espacios se programaron contenidos para los niños (franja mediodía); series para los jóvenes (franja tarde y acceso a prime time) y el informativo general (acceso a prime time).

En relación a la información, en 2006 se emitía un solo noticiario nacional de carácter generalista al día. Sin embargo, se programa por la noche (22.00 horas) y con el cambio se desplazó a la franja de tarde (20.30 horas). Al cambio de horario llevado a cabo por la Corporación se añadió La 2 Noticias Exprés. Una versión resumida del noticiario que se ofreció de lunes a viernes en el late night y en el prime time los fines de semana. Se trataba de franjas hasta el momento carentes de espacios de información de actualidad y cuya incorporación respondía a la táctica de incrementar el tiempo destinado a noticias diarias.

Destaca también la reestructuración de la oferta para el público infantil y juvenil con el nuevo organismo. Con el Ente se dedicaban tres franjas horarias al público infantil pero los protagonistas de todas ellas eran Los Lunnis. El único espacio que no se emitía dentro de este contenedor era la serie japonesa Oliver y Benji en la franja vespertina. En la temporada del cambio, Los Lunnis se programaron solo por las mañanas. Destacando así una oferta para preescolares en la franja matinal, una para escolares en el mediodía y una para niños y jóvenes por la tarde. De manera que se distribuyeron los contenidos atendiendo a las diferentes edades del público infantil y juvenil.

Por último, la programación del fin de semana es prácticamente la misma. Especialmente, en el caso de los contenidos matinales. La cadena se posiciona por la continuidad y permanencia de programas propios que la definen, como son los de carácter religioso los domingos, los musicales e informativos especializados los sábados, y los documentales y deportivos los dos días.

\subsection{Naturaleza de los contenidos}

Se ha analizado el tiempo destinado por la segunda cadena pública a programar espacios informativos, de entretenimiento y formativos. Por un lado de lunes a viernes, y por otro, durante el fin de semana. Elección que se debe a que la cadena presenta una oferta diferenciada los días laborables de los de descanso?

\subsubsection{Tipos de programas de lunes a viernes}

En 2006, la programación semanal se caracterizaba por la oferta de entretenimiento, al representar estos programas el $57 \%$ del tiempo total de emisión diaria (Gráfico 2). El segundo tipo de contenidos con mayor presencia en esta temporada eran los informativos (22\%) y por último, los formativos $(21 \%)$.

Al contrastar estos datos con los de junio de 2007 (período de renovación de la cadena), se observa que con el objetivo de hacer más competitiva La 2, el tiempo 
destinado a programar espacios de entretenimiento aumentó en un 12\% (Gráfico 3). Por el contrario, la ocupación de los espacios informativos se redujo en un $11 \%$. La aportación de los contenidos formativos es un $1 \%$ menor.

Con la Corporación se dedica casi el doble de tiempo a programar espacios formativos sobre los informativos (20\% frente a un $11 \%)$. Un año antes de los cambios tanto la información como la formación tenían un peso importante en la programación de la pública, aunque los espacios informativos contaban con un $1 \%$ más de peso.

Gráfico 2. Ocupación de los distintos tipos de programas que componen la programación de La 2 de lunes a viernes (2006)

\section{Ente Público RTVE}

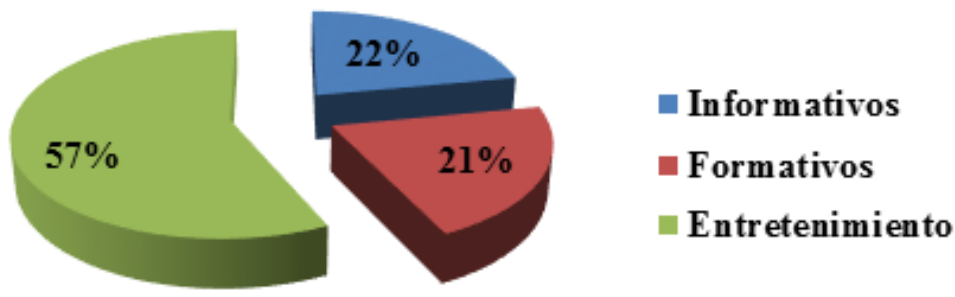

Fuente: Elaboración propia

Gráfico 3. Ocupación de los distintos tipos de programas que componen la programación de La 2 de lunes a viernes (2007)

\section{Corporación RTVE}

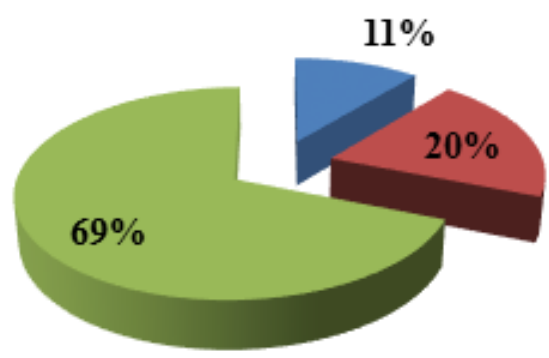

Informativos

- Formativos

Entretenimiento 


\subsubsection{Tipos de programas en el fin de semana}

En cuanto a la programación del fin de semana, la distribución en las dos temporadas es muy similar a pesar de la reestructuración de la cadena. Lo cual es comprensible teniendo en cuenta que la mayoría de los programas que se emiten los sábados y los domingos de junio de 2007 son los que ya se ofertaban antes de acometer la reforma.

Gráfico 4. Ocupación de los distintos tipos de programas que componen la programación de La 2 en el fin de semana (2006)

\section{Ente Público RTVE}

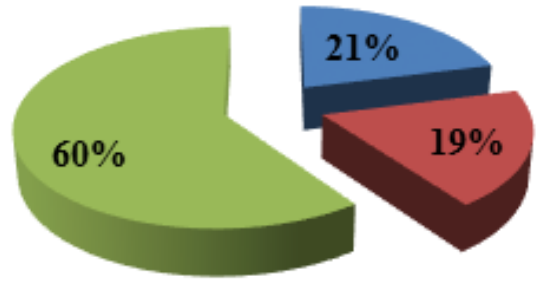

Informativos

- Formativos

Entretenimiento

Fuente: Elaboración propia

Gráfico 5. Ocupación de los distintos tipos de programas que componen la programación de La 2 en el fin de semana (2007)

\section{Corporación RTVE}

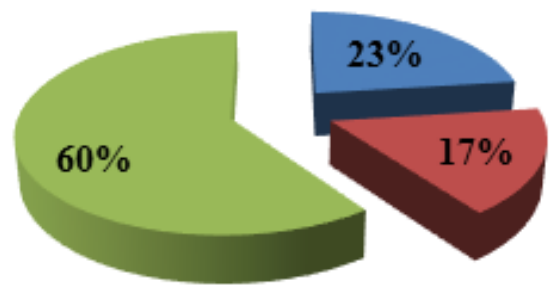

n Informativos

- Formativos

Entretenimiento

Fuente: Elaboración propia 
El principal cambio se basa en la distribución de los tipos de programas que componen la parrilla. Así, el entretenimiento, la información y la formación tienen el mismo peso tanto de lunes a viernes que los fines de semana durante la programación primaveral de La 2 en junio de 2006. Es decir, bajo el mandato del Ente la segunda cadena entretiene, informa y por último, forma los días laborables como los de descanso (Gráfico 4). Por el contrario, con la Corporación la última finalidad del segundo canal de lunes a viernes es informar. Lo cual se convierte en la segunda prioridad durante el fin de semana (Gráfico 5).

\subsubsection{Tipos de programas de entretenimiento}

Las dos parrillas comparten el hecho de que el entretenimiento sea la base de los contenidos de La 2. En un canal de servicio público los espacios informativos y formativos deben ser una pieza fundamental de la parrilla. Por este motivo se ha procedido a analizar los tipos de programas que conforman esta clasificación tanto bajo el mandato del Ente como el de la Corporación.

Gráfico 6. Evolución de los tipos de programas de entretenimiento programados en La 2 de lunes a domingo (en porcentaje)

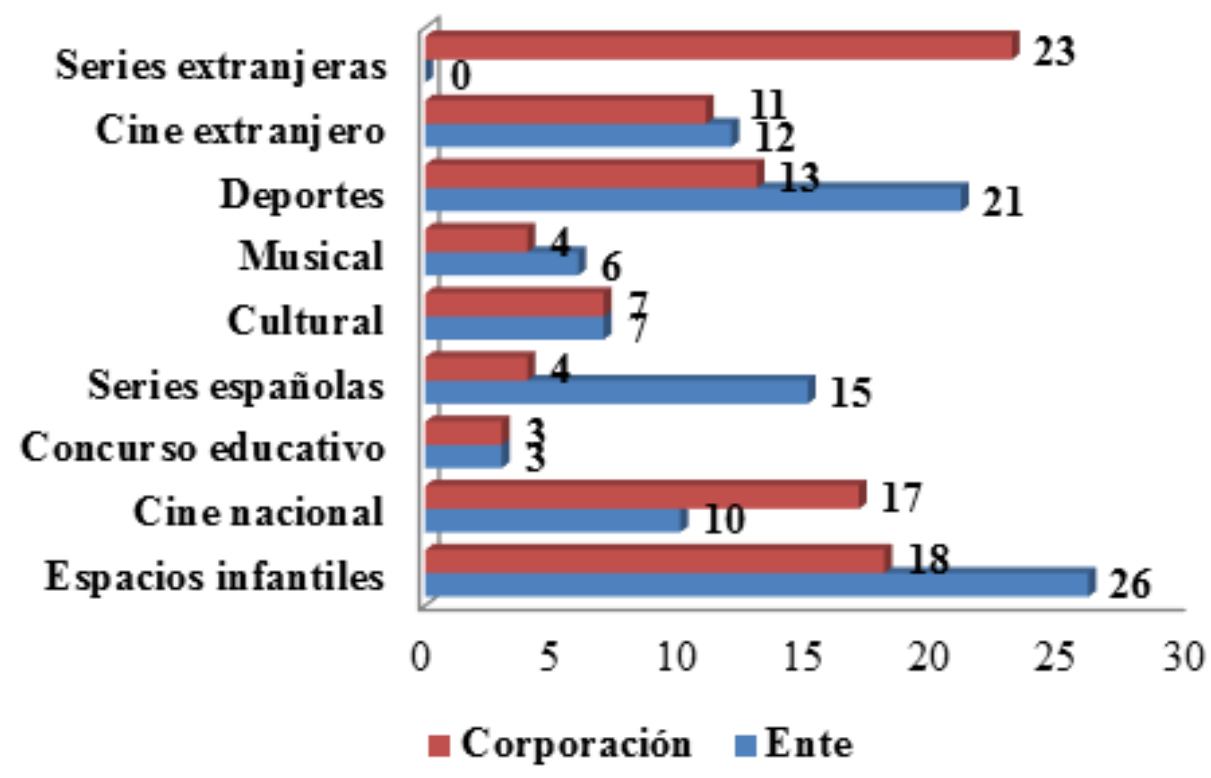

Fuente: Elaboración propia

El entretenimiento constituye una parte indispensable de la programación audiovisual y a diferencia de lo que se suele considerar, abarcan una tipología muy variada de programas. Por lo que además del magacín, los realities, la ficción o el humor 
también comprenden los programas musicales, espacios infantiles, el concurso, los deportes y el cultural (Marín, 2006: 91-98). Y en el caso de La 2, este tipo de espacios contienen elementos que no sólo persiguen entretener al público, sino también transmitirles alguna clase de conocimiento.

Así, al analizar los tipos de programas que componen la oferta de entretenimiento en esta temporada así como el tiempo destinado a cada uno de ellos (Grafico 6), se han encontrado las siguientes particularidades.

En primer lugar, la característica más sobresaliente es cómo en la etapa del Ente Público los espacios infantiles son los programas de entretenimiento que mayor porcentaje representan en la parrilla de la cadena (26\%). Del mismo modo, en esta temporada no se emitió ninguna serie de ficción extranjera que, como se puede ver, constituye la base del entretenimiento en la parrilla del cambio (23\%). Las series internacionales se dirigen principalmente al público juvenil y buscan atraer más audiencia. Además, los espacios infantiles representan el segundo tipo de contenidos de entretenimiento de la Corporación (18\%).

La ficción seriada nacional presenta una elevada ocupación en la etapa del Ente $(15 \%)$. Esto es debido a la programación de la telenovela Obsesión y cuyo cometido no es otro que el de servir de relleno en las franjas de madrugada. Asimismo, se trata de una reposición ya que, su emisión original corresponde al primer canal de TVE.

Por otro lado, los deportes y el musical tienen un peso menor en la nueva programación. Pero se mantienen el cultural y el concurso educativo. Asimismo, el cine nacional se incrementó mientras que, el extranjero, se redujo.

Así, de la comparación de la clase de programas de entretenimiento seleccionados para su emisión en una y otra temporada se observa la dificultad existente para programar espacios que cumplan todos los requisitos que se espera de una cadena pública. Pues vemos como con el cambio de La 2 ganan terreno los espacios dirigidos al público juvenil y aquellos que buscan hacer más competitiva a la cadena pero con el inconveniente de tratarse de ficción seriada extranjera. Sin embargo, se observa un menor porcentaje de tiempo destinado a los deportes y a formatos que deben ser una apuesta segura por parte de la segunda cadena como el musical.

\subsection{Tipo de producción}

Atendiendo al origen de la producción, como se puede ver en el Gráfico 7, en la etapa del Ente Público La 2 se caracterizaba por un gran número de horas dedicadas a la emisión de formatos propios frente a los de producción ajena (58\% frente al $20 \%$ ). Sin embargo, con la reforma de la cadena, el tiempo destinado a programar espacios propios disminuyó significativamente (un 14\% menos de ocupación).

Por lo que en 2006 el carácter de servicio público del segundo canal derivado de la programación de espacios propios es mucho mayor ${ }^{8}$. Predominan los espacios de producción propia frente a los comprados mientras con la Corporación se incrementa 
el pase de programas de producción ajena (adquiridos en el mercado internacional) y externa (encargados a una productora independiente).

Gráfico 7. Ocupación de cada tipo de producción en la programación de La 2 de lunes a domingo (en porcentaje)

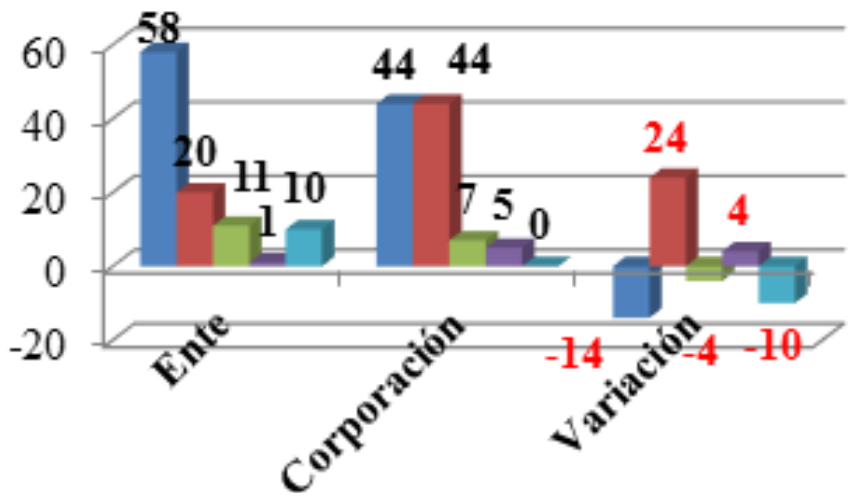

\section{Propia \\ Ajena}

Derechos de emisión

\section{Externa}

Fuente: Elaboración propia

\subsection{Audiencia}

Uno de los principales objetivos de la Corporación fue la recuperación del canal. En el año 2007 La 2 tuvo una cuota de mercado del 4,6\%, por lo que perdió dos décimas respecto al año anterior en el que tenía un $4,8 \%$.

Gráfico 8. Evolución de la audiencia de la televisión generalista en España (20062007)

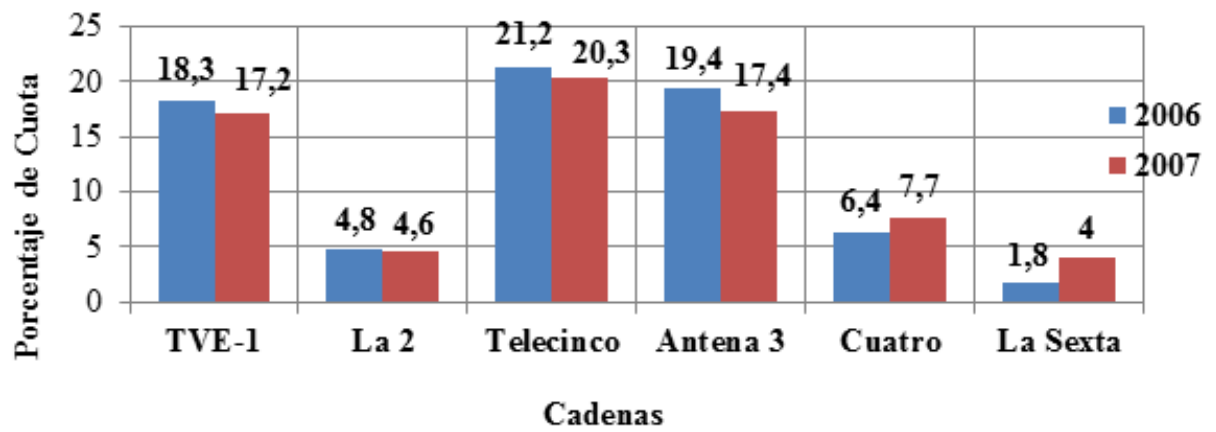

Fuente: Taylor Nelson Sofres Gráfico: Elaboración propia 
Los resultados no son del todo malos si se analizan en relación al contexto del mercado. Como se puede ver en el gráfico 8 , las demás cadenas generalistas perdieron cuota de mercado respecto al ejercicio del año anterior. La Primera pasó de un 18,3\% al $17,2 \%$, lo que supone un descenso de un 1,1\%; Antena 3 del 19,4\% al 17,4\%, una bajada del $2 \%$ y Telecinco, de un $21,2 \%$ en 2006 al 20,3\%, un $0,9 \%$ menos. Sólo los nuevos canales incrementaron su cuota de audiencia en detrimento de los clásicos operadores. Cuatro pasó de un 6,4\% a un 7,7\% y La Sexta, de un 1,8\% el primer año de su difusión, consiguió en otro más alcanzar un $4 \%$ de cuota de mercado.

\section{Conclusiones}

Tras la comparación de las parrillas de programación de La 2 de TVE se concluye que con la Corporación se llevó a cabo una reestructuración de los contenidos de la cadena con el objetivo de hacerla más competitiva. Entre las estrategias de programación que confirman este cambio destaca el comenzar el prime time antes que la competencia; adelantar el inicio del telediario; reorganizar la oferta para llegar tanto al público infantil como juvenil e incluir formatos de éxito en la franja de máxima audiencia.

En segundo lugar se ha encontrado que, al igual que destacaba Gómez Escalonilla (1998: 234-273), el entretenimiento sigue siendo el pilar fundamental de la programación de La 2. Aunque en una televisión pública deben primar los espacios de carácter informativo y formativo, los contenidos con mayor representación son los lúdicos. Pero se trata de una oferta compuesta por series extranjeras para jóvenes, dibujos animados, espacios deportivos, culturales y cine nacional. De manera que, muchos de los espacios que se han clasificado dentro del género de programa de entretenimiento, contienen elementos educativos.

En el intento de ganar audiencia se han incorporado series reconocidas a nivel mundial en la oferta semanal y se han ofrecido contenidos para un espectador no contemplado con el Ente: la juventud. Pero para ello se ha reducido la emisión de programas de servicio público. De manera que, a pesar del cambio del Ente Público a la Corporación RTVE, a la segunda cadena le ha quedado pendiente el desafío de complacer todas las demandas, conciliando información, entretenimiento y educación.

Por último, aunque el nuevo organismo no consiguió frenar la caída de audiencia de la cadena, La 2 de TVE fue de las cadenas generalistas más antiguas que menos cuota de mercado perdió $(0,2 \%)$. 


\section{Referencias bibliográficas}

CONTRERAS, J. M. y PALACIO, M. (2003) La programación de televisión. Madrid: Editorial Síntesis.

CORTÉS, J.A (2001). La estrategia de la seducción. La programación en la neotelevisión (segunda edición). Pamplona: Universidad de Navarra.

GABINETE DE PRENSA DE RTVE (2007). "La 2 se mueve" [en línea]. Dossier de prensa del 13 de abril. Disponible en: http://www.rtve.es. (sala de prensa). [21/09/2013].

GÓMEZ-ESCALONILLA MORENO, Gloria (1998). La programación televisiva en España. Estudio de las parrillas de programación televisiva española desde 1956 a 1996. Madrid: Universidad Complutense de Madrid.

INSTITUTO OFICIAL DE RADIO Y TELEVISIÓN (2007). Corporación RTVE. Principios básicos de la programación. Madrid: Consejo de Administración de RTVE.

LÓPEZ CEPEDA, A. M" (2012). "La incidencia de la Ley 7/2010, General de la Comunicación Audiovisual, en el cambio normativo de Radio Televisión de Galicia”. En: Derecom, n⿳9 9 , págs. 19-34.

LÓPEZ VIDALES, N.; AZURMENDI ADARRAGA, A.; ORTIZ SOBRINO, M.A. (2012). "Hacia un nuevo marco regulatorio y de gestión de la televisión pública en España: el bienio del cambio". En: Revista de la $S E E C I$, año 15, n² 27, págs. 39-59.

MARÍN, C. (2001). Periodismo Audiovisual: información, entretenimiento y tecnologías multimedia. Barcelona: Gedisa.

TAYLOR NELSON SOFRES (2008). Anuario de Audiencias de televisión 2007. Madrid: Sofres Audiencia de Medios.

\section{Notas}

1. El lunes, 31 de agosto de 2009, se publicó en el Boletín Oficial del Estado (núm.210, p.74003) la Ley 8/2009, de 28 de agosto, de financiación de la Corporación de Radio y Televisión Española que entró en vigor el 1 de enero de 2010.

2. Se ha confeccionado el corpus de análisis a partir de los datos de programación publicados por El País y la información proporcionada por el Fondo Documental de Radio Televisión Española correspondiente a las fechas objeto de estudio.

3. La muestra final objeto de estudio corresponde a las siguientes fechas: semana del 12 al 18 de junio de 2006 y semana del 11 al 17 de junio de 2007.

4. Siglas del inglés Ultra High Frequency que significan 'Frecuencia Ultra Alta'.

5. El 15 de enero de 2007, los doce miembros del Consejo de Administración de la Corporación tomaron posesión de sus cargos en el Congreso de los Diputados. Asimismo, el periodista Luis Fernández se convirtió en el primer máximo responsable de RTVE designado por el Parlamento y no por el Gobierno, como había sucedido históricamente. 
6. Remodelación de la programación de La 2" en www.tutele.net [en línea]. 22 de marzo 2007. [02/03/2009].

7. A la hora de cuantificar el tiempo dedicado a cada tipo de programación se incluyen las pausas publicitarias.

8. Hay que precisar que algunos espacios contenedores se han considerado como de producción propia porque el formato es resultado de la producción de la cadena, pero el contenido se basa en espacios de producción ajena o derechos de emisión. Este es el caso de Los Lunnis, de Estadio 2, así como de los contenedores de cine Versión Española y Off Cinema. Para el análisis de la parrilla de 2007 también se contabilizaron como de producción propia.

\section{La autora}

Erika Fernández es Doctora Europea en Comunicación por la Universidad de Vigo. Trabaja como profesora Ayudante Doctor de la Universidad Internacional de la Rioja (UNIR). Imparte las asignaturas de Programación Radiofónica y Televisiva, Creatividad Publicitaria Básica y Diseño y Edición. Es coautora de Las mujeres en la ficción televisiva española de prime time (2011) y La imagen de la crisis económica en los informativos de televisión (2012). 